\title{
High IncRNA MEG3 expression is associated with high mortality rates in patients with sepsis and increased lipopolysaccharide-induced renal epithelial cell and cardiomyocyte apoptosis
}

\author{
KANG CHEN, XIAOJUN SHI, YE JIN, FENG WANG, \\ QING SHEN and WEIMING XU \\ Department of Emergency Medicine, Shanghai Jiao Tong University Affiliated Sixth People's Hospital, \\ Shanghai 201400, P.R. China
}

Received November 8, 2018; Accepted June 13, 2019

DOI: $10.3892 /$ etm.2019.8049

\begin{abstract}
Long non-coding RNA (lncRNA) maternally expressed 3 (MEG3) has been reported to be a key component in cancer biology. Preliminary microarray data from a previous study indicated that lncRNA MEG3 expression was altered in plasma of patients with sepsis. The present study found that the plasma levels of lncRNA MEG3 were significantly higher in sepsis patients compared with healthy controls. Patients were subsequently divided into high and low lncRNA MEG3 expression groups according to the Youden's index. Patients from the high lncRNA MEG3 expression group exhibited a significantly higher mortality rate compared with those from the low lncRNA MEG3 expression group. According to treatment outcomes, patients were divided into survival and mortality groups. It was observed that the pre-therapy expression levels of lncRNA MEG3 were significantly higher in mortality group compared with the survival group. Cell experiments in vitro revealed that lncRNA MEG3 overexpression and silencing using siRNA promoted and inhibited renal epithelial cell and cardiomyocyte apoptosis induced by lipopolysaccharide, respectively. In conclusion, these data suggest that lncRNA MEG3 overexpression may be involved in sepsis, and the downregulation of lncRNA MEG3 may serve as a potential therapeutic target for sepsis.
\end{abstract}

Correspondence to: Dr Xiaojun Shi, Department of Emergency Medicine, Shanghai Jiao Tong University Affiliated Sixth People's Hospital, South Campus, 6600 Nanfeng Highway, Nanqiao New Town, Fengxian, Shanghai 201400, P.R. China

E-mail: gz86962@163.com

Key words: sepsis, long non-coding RNA maternally expressed 3, renal epithelial cell, cardiomyocytes

\section{Introduction}

Sepsis as a systemic inflammatory response syndrome that is caused by severe infections (1). Patients with sepsis usually suffer from insufficient blood flow and poor organ function, resulting in organ failure, tissue hypoperfusion and mortality $(2,3)$. It has been reported that the prevalence of sepsis will be significantly increased in the near future due to increased exposure to a number of risk factors, such as systemic inflammatory diseases (4). In spite of efforts made to improve sepsis therapeutic interventions, treatment outcomes remain unsatisfactory. It is estimated that $30-70 \%$ patients will succumb to sepsis during hospitalization and this unacceptable high mortality rate did not decrease significantly during the past several years $(5,6)$.

Long non-coding RNAs (IncRNAs) are a group of non-protein coding RNAs composed of $>200$ nucleotides (7). In spite of the lack of protein-coding ability, lncRNAs directly participate in physiological and pathological processes (8). The involvement of lncRNAs in sepsis has also been reported by previous studies, and certain lncRNAs are considered good diagnostic markers or therapeutic targets for sepsis $(9,10)$. In particular, the lncRNA maternally expressed 3 (MEG3) is a well characterized lncRNA in cancer biology $(11,12)$. Preliminary microarray data from our study suggested that lncRNA MEG3 expression was also altered in the plasma of patients with sepsis, indicating its involvement in this disease. The present study revealed that IncRNA MEG3 overexpression may be involved in sepsis, and the downregulation of lncRNA MEG3 may serve as a potential therapeutic target for sepsis.

\section{Materials and methods}

Specimens and cell lines. Plasma samples were obtained before treatment from 82 patients with sepsis (sex, 45 males and 37 females; age range, 28.3-69.5 years; mean age, $49.1 \pm 6.1$ years) who were admitted to the Shanghai Jiaotong University Affiliated Sixth People's Hospital South Campus 
(Shanghai, China) from March 2016 to March 2018. Inclusion criteria: i) Patients with sepsis with complete medical records including history of previous diseases and treatment; and ii) patients understood the experimental protocol and signed informed consent. Exclusion criteria: i) Patients additively afflicted with other diseases, such as cancer and metabolic diseases; and ii) patients who were treated by any therapies prior to admission. This study also included 54 healthy controls (sex, 30 males and 24 females; age range, 29.1-66.2 years; mean age, $48.4 \pm 5.6$ years) from Shanghai Jiaotong University Affiliated Sixth People's Hospital South Campus recruited during the same time period. All healthy controls also signed informed consent. This study was approved by the Ethics Committee of Shanghai Jiaotong University Affiliated Sixth People's Hospital South Campus (Shanghai, China). All patients were followed-up for 6 months after admission to record their survival.

Human primary renal mixed epithelial cells $\left(\right.$ ATCC $^{\circledR}$ PCS-400-012 ${ }^{\mathrm{TM}}$ ) were purchased from American Type Culture Collection. The AC16 human cardiomyocyte cell line was purchased from Sigma-Aldrich (Merck KGaA). Cells were cultivated in DMEM medium (Sigma-Aldrich; Merck KGaA) containing 10\% FBS (Sigma-Aldrich; Merck KGaA) at $37^{\circ} \mathrm{C}$ with $5 \% \mathrm{CO}_{2}$.

Reverse transcription-quantitative PCR (RT-qPCR). Total RNA was extracted from plamsa, A16 cardiomyocytes and renal mixed epithelial cells using Monarch ${ }^{\circledR}$ Total RNA Miniprep kit (New England BioLabs, Inc.) according to manufacturer's protocol, followed by reverse transcription using SuperScript ${ }^{\mathrm{TM}}$ III Reverse Transcriptase (Thermo Fisher Scientific., lnc.) and preparation of qPCR reactions using SYBR $^{\circledast}$ Green Quantitative RT-qPCR kit (Sigma-Aldrich; Merck KGaA), according to the manufacturers' protocols. Primers of 1 ncRNA MEG3 and $\beta$-actin endogous control were designed and synthesized by Shanghai GenePharma Co., Ltd. The following primers were used: 1ncRNA MEG3 forward, 5'-CTGCCCATCTACACCTCACG-3' and reverse, 5'-CTC TCCGCCGTCTGCGCTAGGGGCT-3'; and $\beta$-actin forward, 5'-GACCTCTATGCCAACACAGT-3' and reverse, 5'-AGT ACTTGCGCTCAGGAGGA-3'. Using the $2^{-\Delta \Delta C q}$ method (13), IncRNA MEG3 expression was normalized to $\beta$-actin endogenous control.

Cell transfection. Vectors (pcDNA3) expressing lncRNA MEG3, empty pcDNA3 vectors, IncRNA MEG3 siRNA (5'-CCTCTTACCAAAAGACTTA-3') and scrambled negative control siRNA (5'-UGUGCAACGUCCGUCGAAGA-3') were designed and synthesized by Sangon Biotech Co., Ltd. Vectors $(10 \mathrm{nM})$ and siRNAs sequences $(40 \mathrm{nM})$ were transfected into renal epithelial cells $\left(1 \times 10^{5}\right.$ cells $\left./ \mathrm{ml}\right)$ and AC16 human cardiomyocytes $\left(1 \times 10^{5}\right.$ cell/ml) using Lipofectamine ${ }^{\circledR} 2000$ reagent according to the manufacturer's protocol. Cells only treated with Lipofectamine 2000 reagent were designated control cells whereas cells transfected with either the empty vector or scrambled negative control siRNA were were designated negative controls cells.

Cell apoptosis assay. Expression of IncRNA MEG3 was detected using RT-qPCR $24 \mathrm{~h}$ after transfection. Cell apoptosis was detected only in cases where the overexpression rate reached $200 \%$ or the knockdown rate reached $50 \% 24 \mathrm{~h}$ post-transfection. Briefly, cells were harvested and cell suspensions at a density of $4 \times 10^{4}$ cells $/ \mathrm{ml}$ were prepared, which were then seeded into a six-well plate with $2 \mathrm{ml} /$ well and treated with lipopolysaccharide (LPS, $10 \mathrm{mg} / \mathrm{l}$; Sigma-Aldrich; Merck KGaA) to induce apoptosis. Cells were then cultured in $37^{\circ} \mathrm{C}$ under $5 \% \mathrm{CO}_{2}$ for $48 \mathrm{~h}$ before digestion with $0.25 \%$ trypsin, followed centrifugation at $1200 \mathrm{x} \mathrm{g}$ for $10 \mathrm{~min}$ at room temperature. The harvested cells were subjected to staining with Annexin V-fluorescein isothiocyanate and propidium iodide using the Annexin V-FITC Apoptosis Staining/Detection kit (cat. no. ab14085; Abcam) according to manufacturer's protocol (Dojindo Molecular Technologies, Inc.). Apoptotic cells were detected and counted using flow cytometry (FCS Express 6 flow cytometry software; De Novo Software).

Statistical analysis. GraphPad Prism software (version 6; GraphPad Software, Inc.) was used for all statistical analyzes. Youden's index was used to determine the cut-off values. All experiments in this study were performed in triplicate and data are presented as the mean \pm standard deviation or rates. Comparison of mortality frequencies was performed using the $\chi^{2}$ test. Comparisons of lncRNA MEG3 expression levels between sepsis patients and healthy controls were performed using unpaired t-test. Comparisons of cell apoptotic rates between 3 groups were performed using one-way ANOVA followed by Tukey's test. $\mathrm{P}<0.05$ was considered to indicate a statistically significant difference.

\section{Results}

Plasma levels of IncRNA MEG3 are significantly higher in sepsis patients compared with healthy controls. Expression of IncRNA MEG3 in plasma samples of patients with sepsis and healthy controls was detected using RT-qPCR (Fig. 1). Compared with healthy controls, plasma levels of lncRNA MEG3 were significantly higher in patients with sepsis $(\mathrm{P}<0.05)$.

High lncRNA MEG3 plasma levels are closely associated with higher mortality rates in sepsis patients. According to Youden's index, patients were allocated into high $(\mathrm{n}=44)$ and low ( $\mathrm{n}=38$ ) lncRNA MEG3 expression groups. A total of 38 patients succumbed during hospitalization, including 29 cases in the high lncRNA MEG3 expression group, accounting for $65.9 \%$ of this group. A total of 9 cases of mortality were observed in the low lncRNA MEG3 expression group, accounting for $23.7 \%$ of this group. Patients from the high IncRNA MEG3 expression group demonstrated significantly higher mortality rates compared with those from the low lncRNA MEG3 expression group $\left(\chi^{2}=14.6201\right.$; $\mathrm{P}<0.01$; Fig. 2).

Higher pre-therapy IncRNA MEG3 plasma levels are associated with higher mortality rates. According to treatment outcomes, patients were divided into the survival $(n=44)$ and mortality $(n=38)$ groups. It was observed that pre-therapy levels of lncRNA MEG3 in the plasma were significantly 


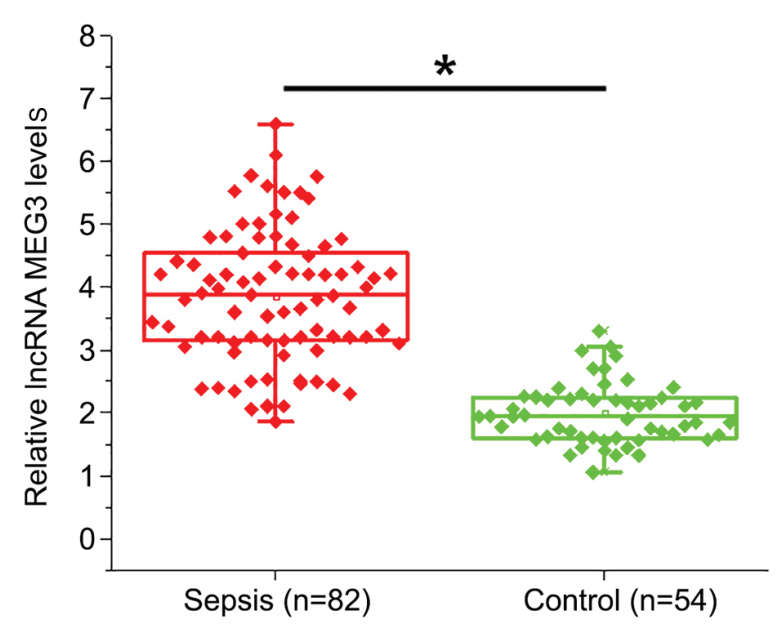

Figure 1. Plasma levels of lncRNA MEG3 were significantly higher in sepsis patients compared with healthy controls. Reverse transcription-quantitative PCR results showed that compared with healthy controls, plasma levels of IncRNA MEG3 were significantly higher in patients with sepsis. ${ }^{*} \mathrm{P}<0.05$. LncRNA, long non-coding RNA; MEG3, maternally expressed 3.

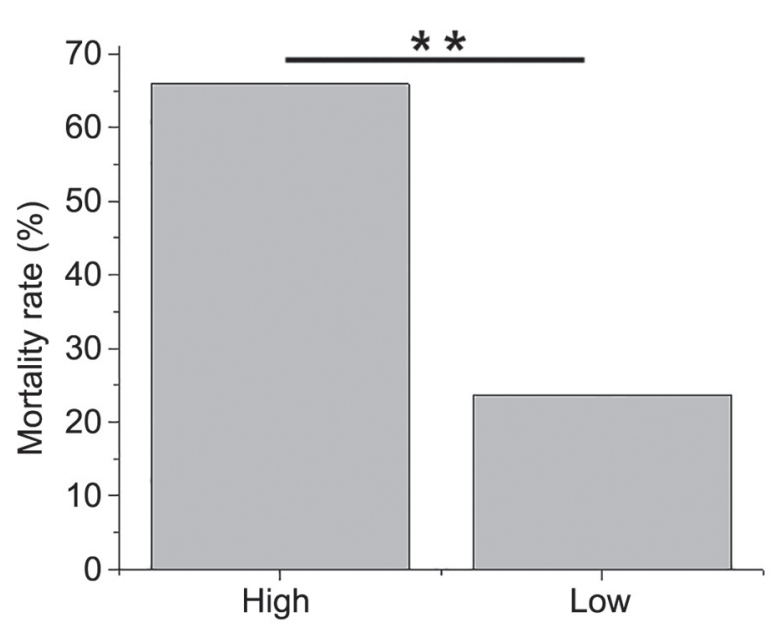

Figure 2. High lncRNA MEG3 levels in the plasma are associated with high mortality rates in sepsis patients. A bar chart comparing mortality rates of patients with sepsis between high and low lncRNA MEG3 expression groups. $\chi^{2}$ test revealed that high lncRNA MEG3 levels in the plasma are associated with high mortality rates in patients with sepsis. $\chi^{2}=14.6201 ;{ }^{* *} \mathrm{P}<0.01$. LncRNA, long non-coding RNA; MEG3, maternally expressed 3; high, high expression group; low, low expression group.

higher in the mortality group compared with the survival group ( $\mathrm{P}<0.05$; Fig. 3$)$.

LncRNA MEG3 regulates renal epithelial cell and cardiomyocyte apoptosis in the presence of lipopolysaccharide (LPS). Cell apoptosis serves an important role in organ failure and death as a result of sepsis (1). Successful knockdown and overexpression of MEG3 were confirmed (Fig. 4A and B). In the presence of LPS treatment, lncRNA MEG3 overexpression and siRNA knockdown resulted in increased and reduced renal epithelial cell (Fig. 4A) and cardiomyocyte (Fig. 4B) apoptosis, respectively, compared with their respective controls $(\mathrm{P}<0.05)$. For renal epithelial cells, MEG3 overexpression increased the

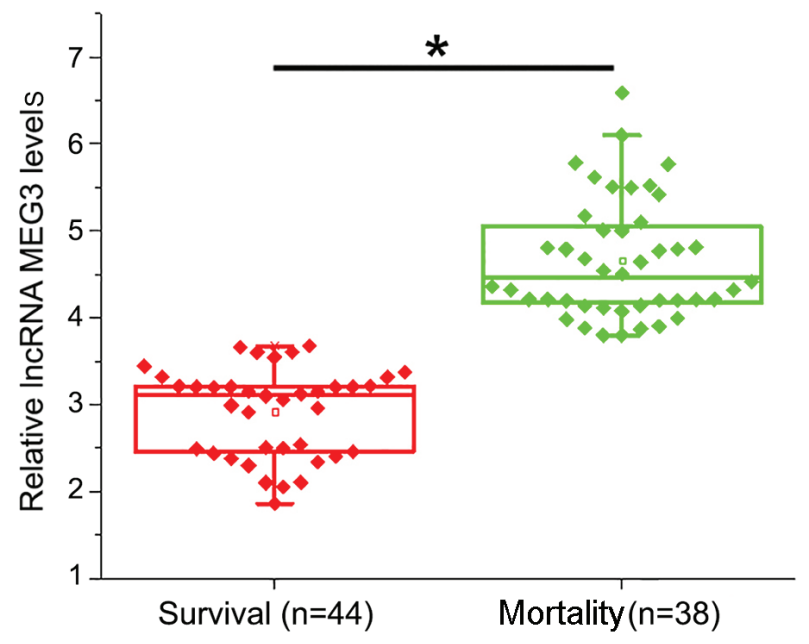

Figure 3. Higher pre-therapy levels of lncRNA MEG3 are observed among patients who succumbed compared with patients who survived. Pre-therapy levels of 1ncRNA MEG3 in the plasma were significantly higher in the mortality group compared with the survival group. ${ }^{*} \mathrm{P}<0.05$. LncRNA, long non-coding RNA; MEG3, maternally expressed 3.

cell apoptotic rate from $\sim 13 \%$ at control to $>20 \%$, whilst MEG3 knockdown decreased the cell apoptotic rate from $\sim 12 \%$ to $<6 \%$ (Fig. 4A). For cardiomyocytes, MEG3 overexpression increased the cell apoptotic rate from $\sim 8 \%$ at control to $>17 \%$, whereas MEG3 knockdown decreased the cell apoptotic rate from $\sim 9 \%$ to $<5 \%$ (Fig. 4B).

\section{Discussion}

The role of MEG3 as a tumor suppressor lncRNA has been well documented in a number of different malignancies $(11,12)$. The key finding of the present study is that lncRNA MEG3 is upregulated during sepsis and downregulation of this lncRNA may serve as a potential therapeutic strategy for this disease by inhibiting apoptosis in different cell types.

Previous microarray studies revealed that the development and progression of sepsis is accompanied by changes in the expression patterns of a large set of lncRNAs (14), suggesting the involvement of lncRNAs in this disease. The differentially expressed lncRNAs promote or inhibit sepsis by participating in the gene interaction network (15). Certain lncRNAs have been proposed as potential biomarkers for sepsis (16). As a tumor suppressor, lncRNA MEG3 is frequently downregulated in a variety of human malignancies, and its overexpression has been demonstrated to inhibit tumor growth and development by affecting physiological processes, including cancer cell proliferation and apoptosis induction (17). The present study found that lncRNA MEG3 expression was upregulated in the plasma samples of sepsis patients compared with healthy controls, and high expression levels of lncRNA MEG3 in the plasma were significantly associated with high mortality rates. Therefore, these observations suggest that pre-therapy levels of plasma lncRNA MEG3 may serve as a potentially useful predictor of poor outcome from sepsis.

The progression of sepsis results in the apoptosis of different cell types $(18,19)$, which in turn leads to multiple organ failure and mortality $(18,19)$. Therefore, development 


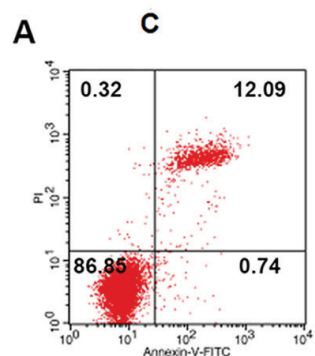

c

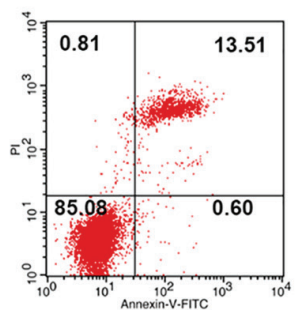

B

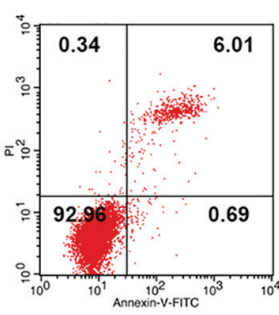

C

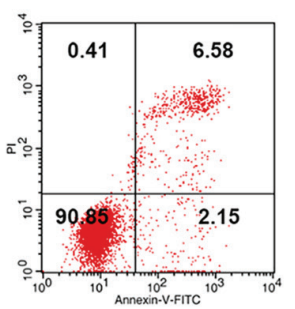

NC

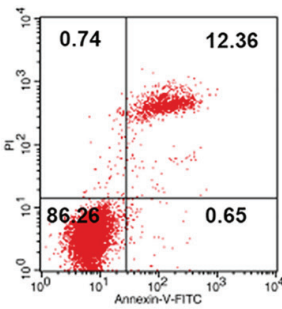

NC

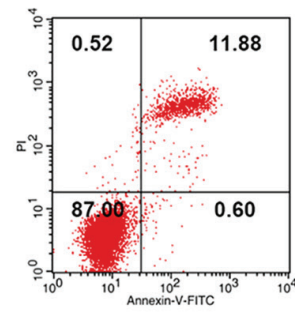

NC

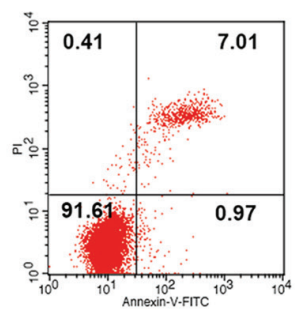

NC

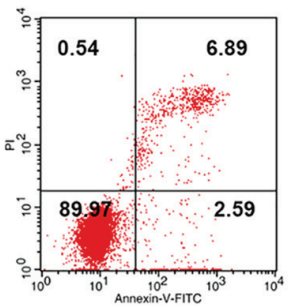

MEG3

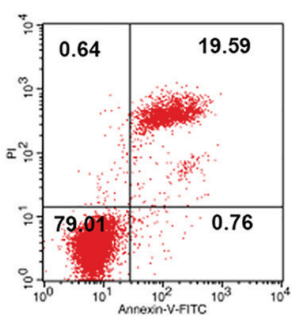

siRNA

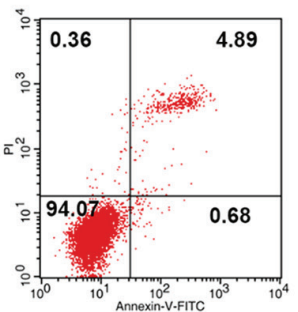

MEG3

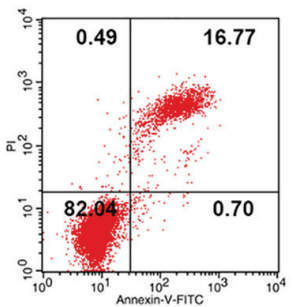

siRNA

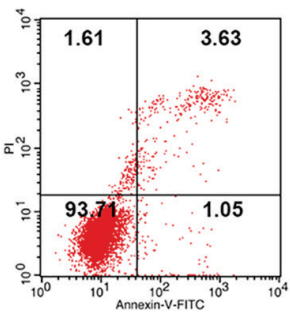

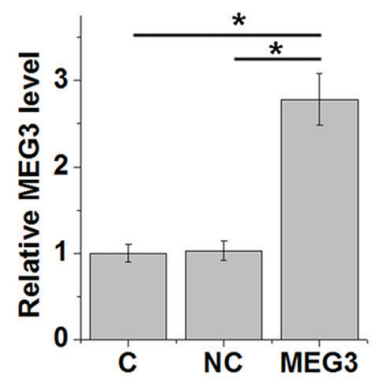
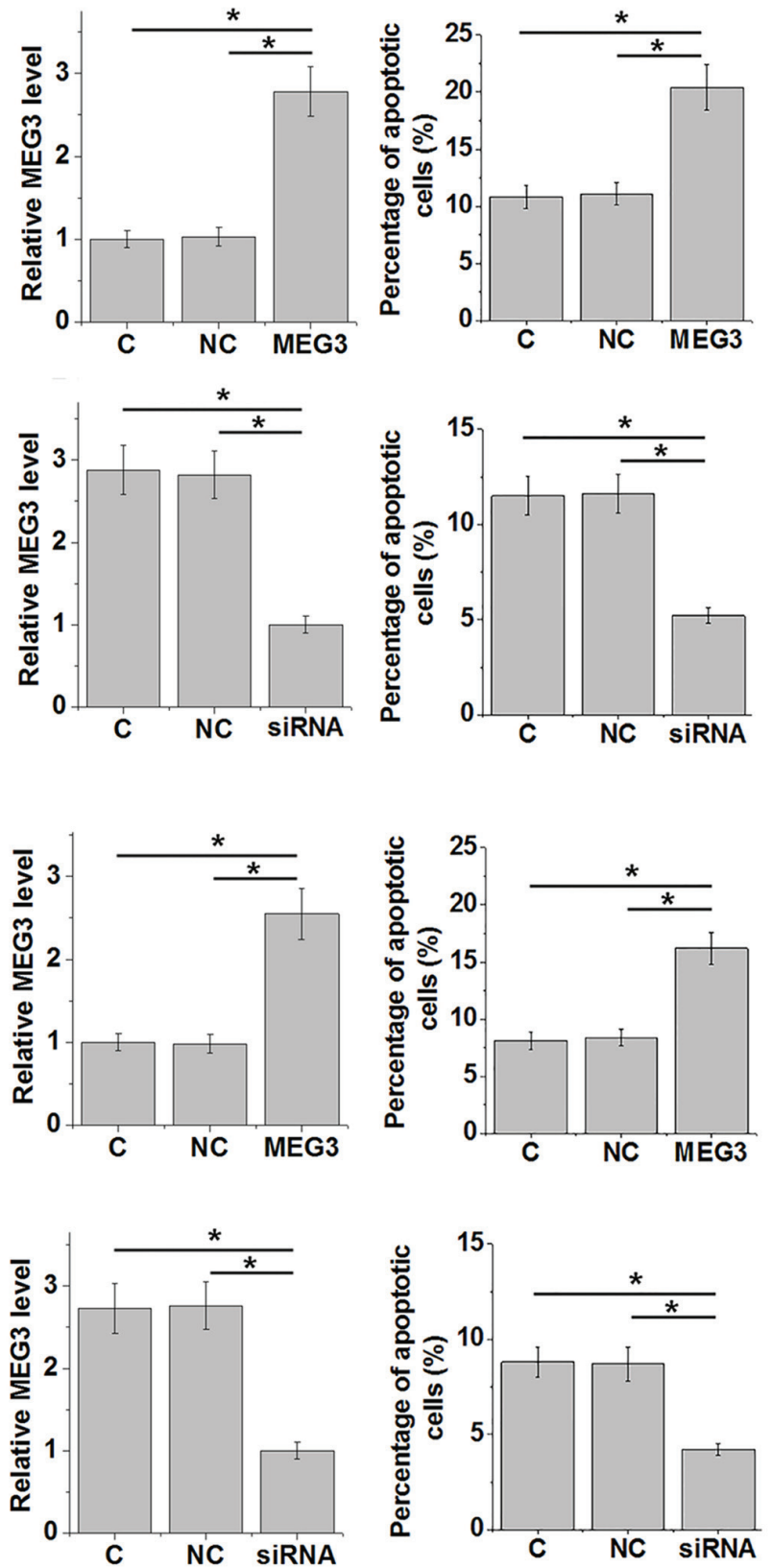

Figure 4. LncRNA MEG3 regulates renal epithelial cell and cardiomyocyte apoptosis following LPS treatment. LncRNA MEG3 overexpression and knockdown were confirmed. Following LPS treatment, LncRNA MEG3 overexpression resulted in significantly increased apoptosis, whereas lncRNA MEG3 knockdown by siRNA significantly inhibited apoptosis in (A) renal epithelial cells and (B) AC12 cardiomyocytes. "P<0.05. LncRNA, long non-coding RNA; MEG3, maternally expressed 3; LPS, lipopolysaccharide.

of therapeutic interventions to inhibit cell apoptosis remains a challenge in the treatment of sepsis. Heart failure and kidney dysfunction are common complications in patients with sepsis (20). In the present study LPS was used to establish a model of sepsis (21). It was found that lncRNA MEG3 overexpression and knockdown increased and inhibited LPS-induced apoptosis of renal epithelial cells and cardiomyocytes, respectively. Therefore, lncRNA MEG3 knockdown may serve as a potential therapeutic target for the treatment of sepsis by inhibiting cell apoptosis in important organs. However, the molecular mechanism of the regulation of cell apoptosis by MEG3 remains unclear, which warrants further study.
Although lncRNA MEG3 exhibited different expression patterns in cancer (downregulation) and sepsis (upregulation) (17), lncRNA MEG3 promotes cell apoptosis in both types of disease, indicating potentially similar functions of lncRNA MEG3 in regulating cell apoptosis across different diseases. Indeed, it has been previously shown that lncRNA MEG3 may interact with p53 to regulate cancer cell apoptosis (17). Therefore, lncRNA MEG3 may be involved in the pathogenesis of sepsis by a similar mechanism.

In conclusion, lncRNA MEG3 is overexpressed in sepsis and downregulation of lncRNA MEG3 may serve as a potential therapeutic target for sepsis by inhibiting cell apoptosis in major organs including the kidneys and the heart. 


\section{Acknowledgements}

Not applicable.

\section{Funding}

No funding was received.

\section{Availability of data and materials}

The datasets used and/or analyzed during the present study are available from the corresponding author on reasonable request.

\section{Authors' contributions}

XS designed experiments. KC, XS and YJ performed experiments. FW, QS and WX collected and analyzed data. XS drafted the paper and all authors approved the final manuscript.

\section{Ethics approval and consent to participate}

This study was approved by the Ethics Committee of Shanghai Jiaotong University affiliated Sixth People's Hospital South Campus (Shanghai, China). Individuals provided informed consent for participation in the present study.

\section{Patient consent for publication}

Patients signed informed consent for potential publication of the present paper.

\section{Competing interests}

The authors declare that they have no competing interests.

\section{References}

1. Martin GS: Sepsis, severe sepsis and septic shock: Changes in incidence, pathogens and outcomes. Expert Rev Anti Infect Ther 10: 701-706, 2012.

2. Liu V, Escobar GJ, Greene JD, Soule J, Whippy A, Angus DC and Iwashyna TJ: Hospital deaths in patients with sepsis from 2 independent cohorts. JAMA 312: 90-92, 2014.

3. Seymour CW, Rea TD, Kahn JM, Walkey AJ, Yealy DM and Angus DC: Severe sepsis in pre-hospital emergency care: Analysis of incidence, care, and outcome. Am J Respir Crit Care Med 186: 1264-1271, 2012.

4. Micek ST, Hampton N and Kollef M: Risk factors and outcomes for ineffective empiric treatment of sepsis caused by gram-negative pathogens: Stratification by onset of infection. Antimicrob Agents Chemother 62: e00007-e000018, 2018.
5. Conde KAP, Silva E, Silva CO, Ferreira E, Freitas FG, Castro I, Rea-Neto A, Grion CM, Moura AD, Lobo SM, et al: Differences in sepsis treatment and outcomes between public and private hospitals in Brazil: A multicenter observational study. PLoS One 8: e64790, 2013.

6. Fleischmann C, Scherag A, Adhikari NK, Hartog CS, Tsaganos T, Schlattmann P, Angus DC, Reinhart K and International Forum of Acute Care Trialists: Assessment of global incidence and mortality of hospital-treated sepsis. Current estimates and limitations. Am J Respir Crit Care Med 193: 259-272, 2016.

7. Shi X, Sun M, Liu H, Yao Y and Song Y: Long non-coding RNAs: A new frontier in the study of human diseases. Cancer Lett 339: 159-166, 2013.

8. Quinn JJ and Chang HY: Unique features of long non-coding RNA biogenesis and function. Nat Rev Genet 17: 47-62, 2016.

9. Ho J, Chan H, Wong SH, Wang MH, Yu J, Xiao Z, Liu X, Choi G, Leung CC, Wong WT, et al: The involvement of regulatory non-coding RNAs in sepsis: A systematic review. Crit Care 20: 383, 2016.

10. Chen $H$, Wang $X$, Yan $X$, Cheng $X$, He $X$ and Zheng W: LncRNA MALAT1 regulates sepsis-induced cardiac inflammation and dysfunction via interaction with miR-125b and p38 MAPK/NFאB. Int Immunopharmacol 55: 69-76, 2018.

11. Modali SD, Parekh VI, Kebebew E and Agarwal SK: Epigenetic regulation of the lncRNA MEG3 and its target c-MET in pancreatic neuroendocrine tumors. Mol Endocrinol 29: 224-237, 2015.

12. Guo Q, Qian Z, Yan D, Li L and Huang L: LncRNA-MEG3 inhibits cell proliferation of endometrial carcinoma by repressing Notch signaling. Biomed Pharmacother 82: 589-594, 2016.

13. Livak KJ and Schmittgen TD: Analysis of relative gene expression data using real-time quantitative PCR and the 2(-Delta Delta C(T)) method. Methods 25: 402-408, 2001.

14. Shu Q: Differential expression profile of long non-coding RNAs from Gr-1+CD11b+ myeloid cells in response to sepsis in mice (INM2P. 424). J Immunol 192: 56-57, 2014.

15. Pellegrina DVS, Severino P, Barbeiro HV, de Souza HP, Machado MCC, Pinheiro-da-Silva F and Reis EM: Insights into the function of long noncoding RNAs in sepsis revealed by gene co-expression network analysis. Noncoding RNA 3: pii: E5, 2017.

16. Dai Y,Liang Z, Li Y,Li C and Chen L: Circulating Long Noncoding RNAs as Potential Biomarkers of Sepsis: A Preliminary Study. Genet Test Mol Biomarkers 21: 649-657, 2017.

17. Lu KH, Li W, Liu XH, Sun M, Zhang ML, Wu WQ, Xie WP and Hou YY: Long non-coding RNA MEG3 inhibits NSCLC cells proliferation and induces apoptosis by affecting p53 expression. BMC Cancer 13: 461, 2013.

18. Ward PA: Sepsis, apoptosis and complement. Biochem Pharmacol 76: 1383-1388, 2008.

19. Hotchkiss RS and Karl IE: Endothelial cell apoptosis in sepsis: A case of habeas corpus? Crit Care Med 32: 901-902, 2004.

20. Schefold JC, Filippatos G, Hasenfuss G, Anker SD and von Haehling S: Heart failure and kidney dysfunction: Epidemiology, mechanisms and management. Nat Rev Nephrol 12: 610-623, 2016.

21. Tanaka KA, Kurihara S, Shibakusa T, Chiba Y and Mikami T: Cystine improves survival rates in a LPS-induced sepsis mouse model. Clin Nutr 34: 1159-1165, 2015.

This work is licensed under a Creative Commons Attribution-NonCommercial-NoDerivatives 4.0 International (CC BY-NC-ND 4.0) License. 